\title{
Computer-based cognitive rehabilitation: the CoRe system
}

Anna Alloni (1) ${ }^{1}$, Elena Sinforiani (2) , Chiara Zucchella (2), Giorgio Sandrini (2) , Sara Bernini (2), Barbara Cattani (3) , Daniela Tost Pardell (4), Silvana Quaglini (1) , and Caterina Pistarini (3).

(1)Department of Electrical, Computer and Biomedical Engineering, University of Pavia, Pavia, Italy,

(2) IRRCS C. Mondino Foundation, Pavia, Italy,

(3) IRCCS S. Maugeri Foundation, Pavia, Italy,

(4) Computer Graphics Division CREB, Universitat Politècnica de Catalunya, Barcelona, Spain

\section{ABSTRACT ARTICLE HISTORY}

Purpose: This work aims at providing a tool for supporting cognitive rehabilitation. This is a wide field, that includes a variety of diseases and related clinical pictures; for this reason the need arises to have a tool available that overcomes the difficulties entailed by what currently is the most common approach, that is, the so-called pen and paper rehabilitation. Methods: We first organized a big number of stimuli in an ontology that represents concepts, attributes and a set of relationships among concepts. Stimuli may be words, sounds, 2D and 3D images. Then, we developed an engine that automatically generates exercises by exploiting that ontology. The design of exercises has been carried on in synergy with neuropsychologists and speech therapists. Solutions have been devised aimed at personalizing the exercises according to both patients' preferences and performance.

Results: Exercises addressed to rehabilitation of executive functions and aphasia-related diseases have been implemented. The system has been tested on both healthy volunteers (n $1 / 4$ 38) and patients (n $1 / 4$ 9), obtaining a favourable rating and suggestions for improvements.

Conclusions: We created a tool able to automate the execution of cognitive rehabilitation tasks. We hope the variety and personalization of exercises will allow to increase compliance, particularly from elderly people, usually neither familiar with technology nor particularly willing to rely on it. The next step involves the creation of a telerehabilitation tool, to allow therapy sessions to be undergone from home, thus guaranteeing continuity of care and advantages in terms of time and costs for the patients and the National Healthcare System (NHS)

\section{IMPLICATIONS FOR REHABILITATION}

- Cognitive impairments can greatly impact an individual's existence, appreciably reducing his abilities and autonomy, as well as sensibly lowering his quality of life. Cognitive rehabilitation can be used to restore lost brain function or slow down degenerative diseases.

- Computerization of rehabilitation entails many advantages, but patients - especially elderlypeople - might be less prone to the use of technology and consequently reluctant towards this innovative therapeutic approach.

- Our software system, CoRe, supports a therapist during the administration of rehabilitation sessions: exercises can be generated dynamically, thus reducing repetitivity, and patients' performance trends automatically analysed to facilitate the assessment of their progress.

1 CONTACT Anna Alloni, e-mail: anna.alloni@unipv.it, address: Department of Electrical, Computer and Biomedical Engineering, University of Pavia, via ferrata, 5 - 27100, Pavia, Italy 
- Tests performed on both healthy subjects and patients provided useful information that allowed us to define an implementation strategy able to reduce patients' resistance to computerized rehabilitation as much as possible.

\section{Introduction}

"Cognitive impairment" is actually an umbrella term under which an extremely varied number of medical conditions can be classified. This variety concerns not only the nature of the cerebral deficit causing the impairment itself (a stroke, Parkinson's disease, dementia, traumatic brain injuries, etc.), but also its results. Different brain functions are in fact directly associated with specific cerebral areas: for this reason, a localized trauma (or, in general, any type of localized injury) results in reduction - or, in the worst cases, in complete loss - of specific abilities, while at the same time other skills remain intact.

The concept of rehabilitation in the field of cognitive impairment can be considered a topic of great interest for researchers, and in the latest years many studies have been performed, trying to establish its efficacy [1-7]. It is not easy to scientifically verify this hypothesis, due to the already mentioned variety of clinical conditions: this makes it very hard to find a sample population numerous enough to be statistically significant, and at the same time sufficiently homogeneous for the cases featured to be considered comparable. Besides, this variability of conditions also complicates the situation, making the creation of a standard protocol to be undergone by all the patients indistinctly an almost impossible task. An alternative to randomized clinical trials are observational studies where, in addition to variables related to the treatment, a big number of possible confounders are collected and used to predict the outcome. However, the most common approach to rehabilitation is based on execution of pen-and-paper exercises (that is, assignments printed on paper, to be executed not unlike school homework), thus the management of the patients' data and their interpretation is not an easy task. Keeping track of several parameters and of the chronological progress of a subject is a manual activity that implies going through transcriptions of previous sessions.

Moreover, being the exercises proposed nothing more than standard, pre-printed forms, the number of stimuli and combinations available is limited. This leads

to onset of boredom and the concrete possibility of a decrease in willingness to undergo the therapy (drop of compliance rate).

During the last years, researchers in the field of medical informatics started developing computer-based solutions to overcome those problems. To date, several rehabilitation software products are available for purchase [8-16]. They share some characteristics, for example, the capability of adapting the difficulty level of the exercises to the patients' performance and the possibility of choosing different sets of exercises according to the patients' deficits, but none offers the same advantage our system does, derived from being based on an ontology.

As a matter of fact, the stimuli ontology allows several degrees of personalization (better explained below), and the possibility to generate (in theory) an unlimited number of different exercises. Those features are fundamental to create patient-tailored tasks. Another peculiarity of our system, that is called CoRe (acronym for COgnitive REhabilitation), is to address age-related compliance. In fact, while this kind of applications is in general well-accepted by young people or children, older patients (e.g. post-stroke or with degenerative diseases), which represent the majority of the treated cases, may have some problems with them, since third age population is usually not very familiar with 
technology [17-19]. This often reflects negatively on attempts to integrate such technology into the therapy routine: feeling unprepared when put in front of something new and very likely never seen before, the patients could end up developing some sort of "hostility" towards a tool they are not able to handle with ease.

This paper, which is an extension of [20], describes how CoRe, able to automatically handle the critical aspects of the rehabilitation procedure, tries to overcome the abovementioned problems, thus offering a valuable support to both medical personnel and patients. It also focuses on the test phase undergone by the system: usability tests were in fact performed, whose results are described, on a sample of healthy subjects diverse in age, education level and degree of familiarity with a PC. Being the system's continuous development highly affected by this experiment, as well as from the feedback obtained from a pilot study performed on a small set of patients, after describing the system implementation used within the tests we report on those first users' feedback and highlight the solutions designed (and currently in implementation stage) to overcome problems and issues emerged, as well as to make the system more and more compliant with the needs of its intended users.

\section{Methods}

\section{The system architecture}

As shown in Figure 1, CoRe features four modules:

- the "front end", in form of a graphic user interface (GUI) allowing the therapist to compose personalized treatment plans by selecting the exercises and by setting the parameters needed to generate each exercise (the difficulty level, number and maximum duration of the stimuli to be shown, etc.);

- the storage module, that is two databases. The first one is used to store the patients' personal information (patients' profiles) and performance parameters. The second one stores all the stimuli (texts, sounds and images) made available for the execution of the sessions. It is populated starting from a stimuli ontology, as illustrated in the next section;

- the "back end", a software engine able to generate customized exercises based on the input received from the GUI and, if the therapist chooses the option for automatic difficulty setting, on the patient's performance;

- the exercises: each each of them is an independent sub-module. Most of them are computerized versions of existing pen-and-paper exercises. Others have been created to meet specific requirements made by the therapists and exploit PC functionalities that would be particularly difficult to reproduce with a classic, computer-free, approach. For example, the "Imageand sound" exercise (see chapter 3 for a detailed illustration).

Once the parameters have been set, the session is started; during the dynamic generation of the executable exercise, performance data of the patient are retrieved to determine the proper difficulty level (in case the settings required automatic definition). The other database is accessed to retrieve stimuli that match the requirements. Besides retrieval operations, the databases can also be accessed for updates. While deletion of records is not allowed, it is possible to exclude a stimulus from the array of available elements, just by setting its "inclusion" flag (an attribute common to all the records of the stimuli database) to false through the GUI. 


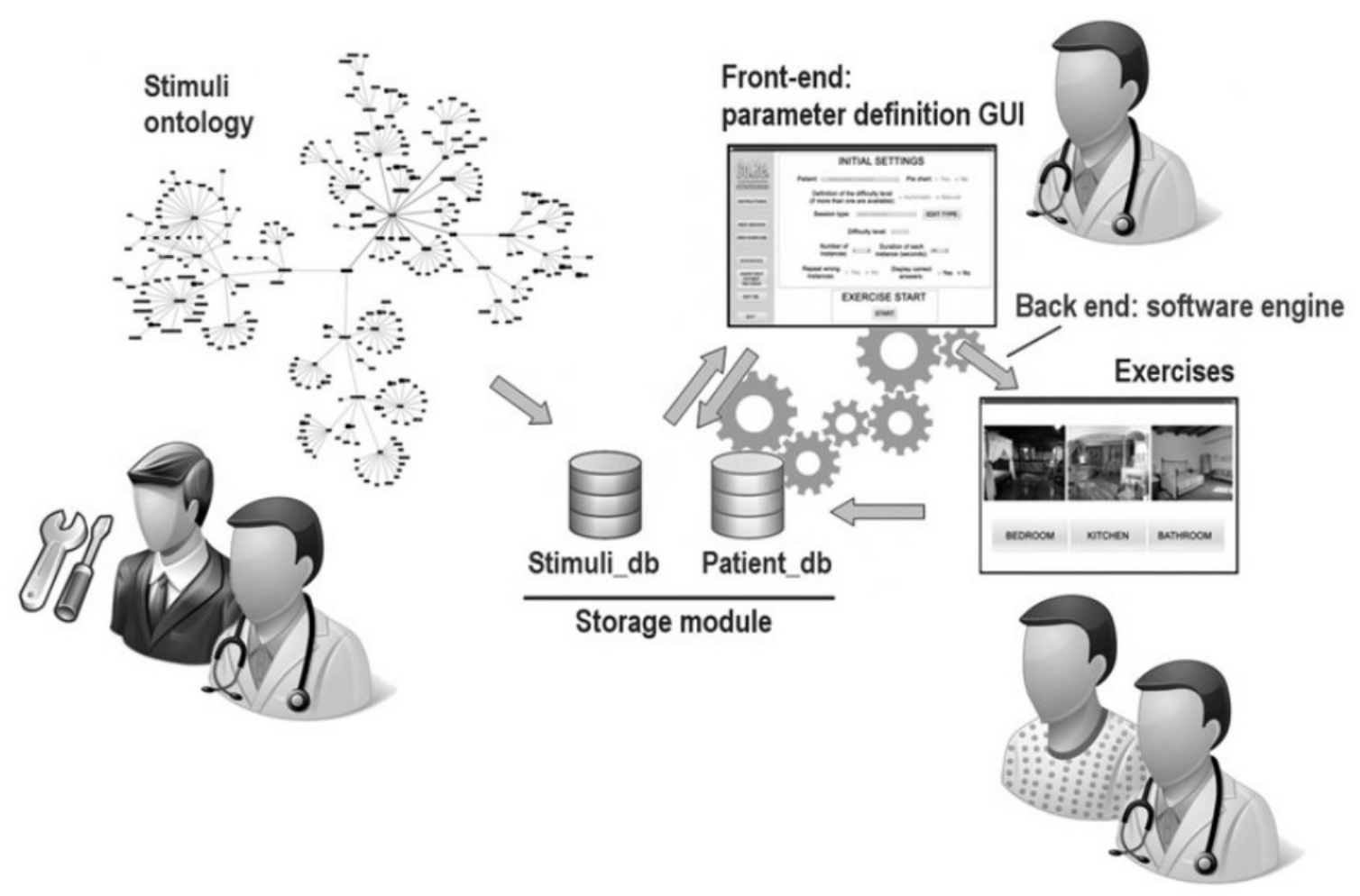

Figure 1: A schema illustrating the structure of CoRe and the intended users of each module: the setup GUI (front-end), meant to be used by the therapist; the exercises, generated by the software engine (back-end) and executed by the patient with the therapist's support. Data are saved in the storage module, based on an ontology created and populated by the developer in collaboration with the therapist.

The great number of stimuli (texts, images and sounds) available in our system allows great variability in execution, something that cannot be achieved with pen and paper exercises. In fact, it is almost impossible for a patient to see multiple instances of a session based on the same stimuli. This helps keeping a high level of attention and involvement, thus reducing the risk of boredom.

\section{The stimuli ontology}

Some of the exercises featured in the system are based on just the correct recognition of the stimuli. proposed - see Figure 2 - while others also require the user to identify relationships defined between stimuli, as seen in Figure 3.

From a technical point of view, the most effective way of building such system - that is, one able to generate relation-based exercises correctly - is by organizing all the stimuli in an ontology, which describes every element through a set of attributes, and its relations with other entities. For example, the attribute "difficulty level" ("low", "medium" and "high") associated with every single concept of the structure and related to the concept itself, may be considered initially to retrieve stimuli according to the patient's scholarship (even if exercises will then be adjusted according to performance data). "Is-a" relations between stimuli can instead be used for classification tasks, while other, user-defined relations can be used to generate several types of exercises, as is the case with the following example, based on the "lives_in" relation:

DOG is related to KENNEL as BIRD is related to?

(a) hole (b) nest (c) house 


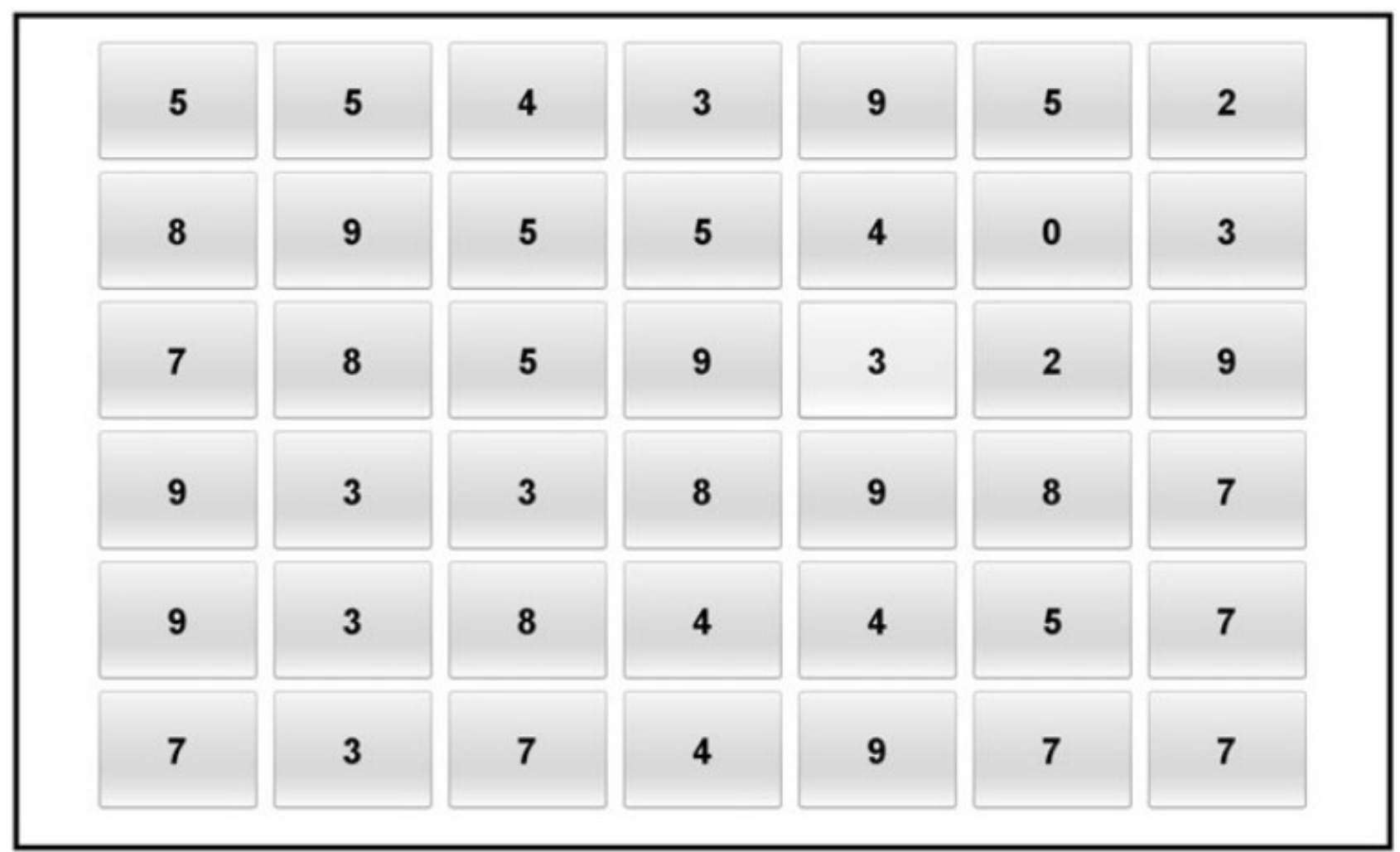

Figure 2. An instance of "Pick the element": the patient is shown a matrix of numbers (textual stimuli) among which he must choose the one requested by the system

A detailed description of the ontology is beyond the scope of this paper and can be found in [21]. Here we only remark, as shown in Figure 1, that the stimuli DB is actually created starting from the ontology. The taxonomy is saved as an XML file whose content (concepts and relations) can be automatically transferred.to the database, thanks to an ad hoc software "translator". Addition of new stimuli or new relationships occurs exclusively through the ontology interface (the ontology has been developed using Prote'ge ), in such a way to guarantee a homogeneous description of all stimuli and to maintain the overall consistency of the stimuli D.

A specific section of the ontology is related to personalization of the exercises. This is a different concept with respect to adaptation of the difficulty of exercises according to the patient's performance. In CoRe, personalization means to replace generic stimuli (e.g. the word "daughter") with stimuli gathered from the patient's life (e.g. his/her daughter "Mary", or a picture of Mary). Privacy issues are taken into account, so that those stimuli will be used only for that patient, unless he gives consent to allow his stimuli becoming part of the generic ontology.

\section{D stimuli}

In recent years, with serious games gaining public attention and becoming an object of interest for researchers, 3D graphics have been introduced in many fields of the medical practice, among which psychological rehabilitation [22-27].

We chose to enrich our system with some 3D-based stimuli because, for a human subject, handling material objects is perceived as more natural than interacting with bare text (the words that "represent" said objects), but being the use of physical items during rehabilitation sessions a clearly non-viable option (at least if several instances of the 
exercise have to be administered), the best alternative is creating virtual models of every object.

The advantages entailed by the use of three-dimensional representations are twofold: first, the use of 3D models allows a more complete interaction, giving users the chance to have a 360 \# view of the objects and, in general, to manipulate them at pleasure: second, the programmer has complete control over the looks of a model (every single vertex, edge and face of a solid is editable in any moment) something that does not happen with 2D images.

In the implementation phase, carried out in collaboration with the Computer Graphics Division of the Polytechnical University of Catalonia (UPC), some important matters had to be taken into consideration. First and most important, a compromise had to be reached between detail and complexity of the models: the more the vertices, the bigger the resulting object file and the computational power needed to render the scene. This will be even more important when the time comes to use CoRe and the re-imagined 3D exercises for telerehabilitation sessions, since our typical patients very likely do not own powerful PCs. Thus, it was necessary to keep the system requirements of our software as low as possible. As a thumb rule, we chose to set the maximum number of vertices-per-object to about 10000.

We used the 3D graphics software Blender (in particular its Game Engine) to develop both the scenarios and all the virtual models to be used within any 3D-based task that will be developed. To date, about 50 models have been created, belonging to 7 different categories (some of which can be seen from Figure 4); the work is not over, others will be generated to enrich the archive as much as possible: this way 3D-based tasks will be allowed the same variability that text/image/ sound-based exercises already show.

\section{Results}

The first implementation of CoRe was aimed at the rehabilitation of executive functions, logical reasoning, strategy, planning, problem-solving and hypothetical- deductive reasoning skills - with particular focus on parkinsonian and post-stroke subjects (which represent the majority of the clinical cases handled by our medical partner, the IRCCS C. Mondino Foundation). We implemented 10 exercises, namely:

- Word coupling: eight words are displayed on screen. The patient must associate them in four couples, identifying the relations that exist between the stimuli;

- Pick the element: a matrix of random text elements (letters or numbers) is displayed. The patient must identify and select the requested element;

- Find the intruder: five words are displayed, four of which belong to the same category. The patient must identify the common category and consequently select the only intruder;

- Unscramble the sentence: scrambled words are displayed. The patient must select them in the right order to form a sentence;

- Unscramble the images: same as above, the patient must put the scrambled images in the right order to form a short story;

- Functional planning: a verb describing an activity is displayed. The patient must select from a list the element related to that activity (e.g. if the action is "writing" and the elements are "candle", "doorknob", "pen" and "notebook", the patient will have to select the latter two); 
- Image and sound: a sound is played, whose duration can be either "long" or "short". Meanwhile an image is displayed, whose dimensions can be "big" or "small". The patient must evaluate whether duration and dimensions "match" (that is, big image + long sound or small image + short sound, according to a criterion explained before the execution) or not;

- Find the category: three images are displayed. The patient must identify the common category to which all of them belong;

- Logical sequences: a logical sequence of elements (numbers or images) with a blanked out element is displayed. The patient must identify, among several options, the correct one to complete the series;

- Logical analogies: the textual version of a mathematical proportion is shown, with one of the four terms blanked out (e.g.: FELINE is related to TIGER as? is related to EAGLE). The patient must identify the relation and therefore select the right element among the proposed options (in the proposed example, given the options FISH and BIRD the patient will select the latter).

The pilot study illustrated in "Test phase and issues" section is related to this set of exercises. Recently, in collaboration with speech therapists from IRCCS S. Maugeri Foundation, another partner in the CoRe project, we started integrating new exercises for aphasic subjects into CoRe. Aphasia is not a disease per se, but a clinical condition resulting from the onset of other diseases. What causes aphasia is an acquired brain damage - most commonly due to head injuries and stroke (25-40\% of the cases), but also cancer, epilepsy and Alzheimer's disease (acute aphasia), or even brain tumours or dementia (progressive aphasia). The consequences of this condition strongly affect a patient's everyday life and hinder his attempts at living a normal life by compromising fundamental functions such as words recalling, understanding, speaking, reading and/ or writing. The types of exercises currently planned (which will be integrated into a new implementation of CoRe and tested on a consecutive set of aphasic patients at the IRCCS S. Maugeri Foundation) are the following:

- Phonemic discrimination: two auditory stimuli are played in sequence and the patient must decide whether they are the same word/syllable;

- Visual/auditory lexical decision: a stimulus is played/shown as a text on screen. The patient must decide whether it is a word or a non-word;

- Comprehension of spoken/written words: a stimulus is played/shown as a text on screen, while four images are displayed: one represents the stimulus, one is a visual/semantic distractor, the others are wrong answers unrelated to the solution The patient must identify the correct image;

- Comprehension of spoken/written sentences: as above, but the stimuli are a description of a scene (the cue), and four images depicting similar situations. The patient must identify the one that represents the scene correctly;

- Discrimination of grammatical correctness of spoken/written sentences: a sentence is spoken/ displayed as text and the patient must decide whether it is grammatically and syntactically correct;

- Recognition of words/non-words (among other words/non-words): 4, 6 or 8 words/non-words are spoken/displayed on screen; the player must recognize the requested one;

- Comprehension of oral/written definition: the definition of an object is spoken/displayed on screen. Four answer options are displayed as text or images. The patient must select the right one;

- Classification: an implementation similar to "3D find the intruder" is planned. An object is shown, together with some boxes, each of which contains some elements 
belonging to a common category. The player must place the object and put it in the correct box;

- Comprehension of spoken/written compound words, according to rules of language;

- Recognition of syllables: a matrix of syllables is shown. The patient must identify the requested one;

- Comprehension of spoken/written paragraphs;

- Daily life-oriented exercises for acalculia: various exercises oriented to management of money (adding amounts, calculating change, etc.). The sum of money to be handled will be represented either as text or images of the banknotes: this way it will also be possible to implement a task of recognition of banknotes (matching the image of the banknote with its value).
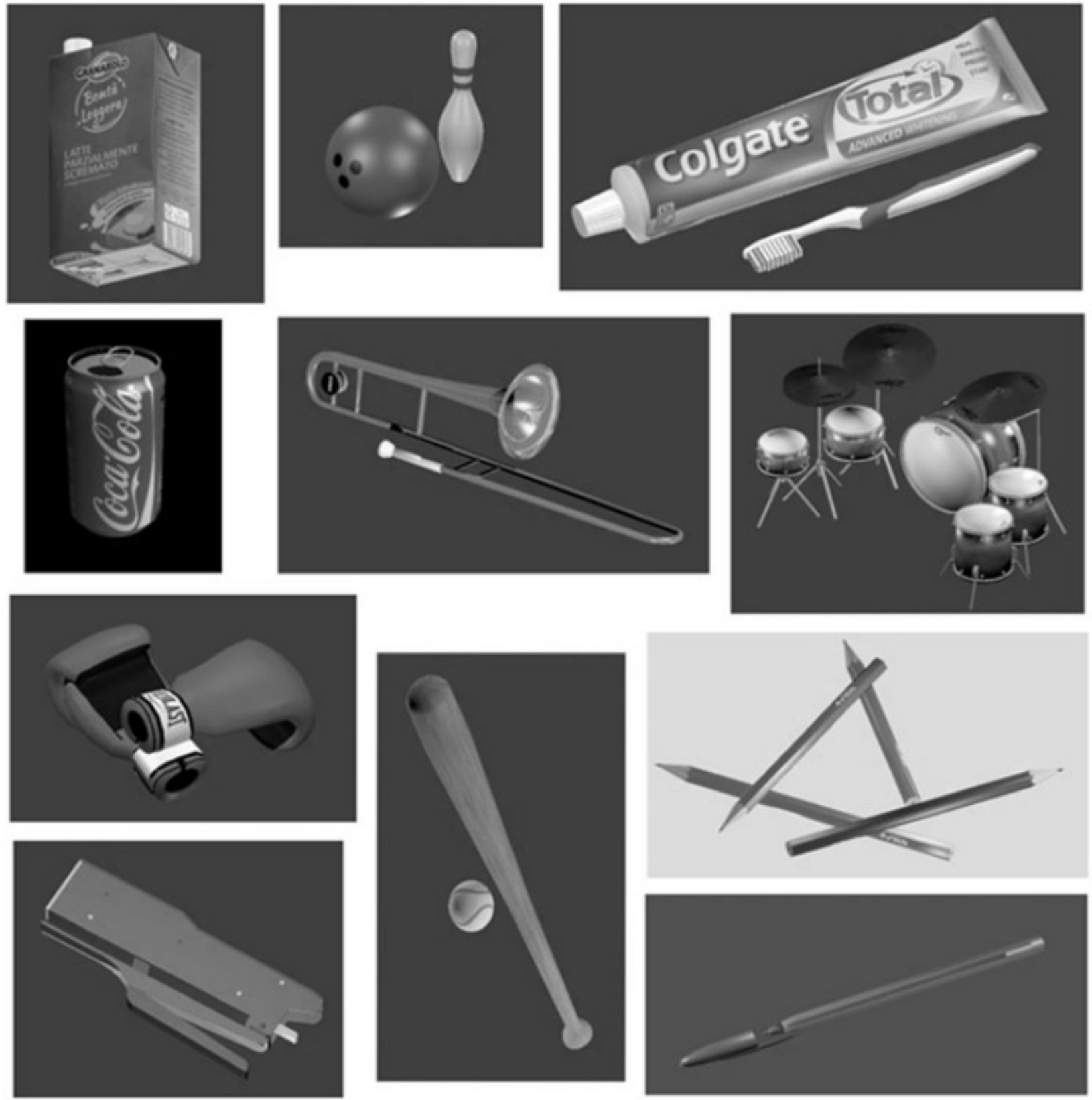

Figure 4. Some of the models created for the 3D exercises. 


\begin{tabular}{|c|c|c|c|c|c|c|}
\hline & & $\begin{array}{l}\text { 1(Totally } \\
\text { disagree) }\end{array}$ & 2 & 3 & 4 & $\begin{array}{c}\text { 5(Totally } \\
\text { agree) }\end{array}$ \\
\hline 1. & I think that I would like to use this system frequently & 0 & 0 & 2 & 9 & 7 \\
\hline 2. & I found the system unnecessarily complex & 14 & 4 & 0 & 0 & 0 \\
\hline 3. & I thought the system was easy to use & 0 & 0 & 4 & 5 & 9 \\
\hline 4. & I think that I would need the support of a technical person to be able to use this system & 9 & 3 & 3 & 2 & 1 \\
\hline 5. & I found the various functions in this system were well integrated & 0 & 0 & 0 & 7 & 11 \\
\hline 6. & I thought there was too much inconsistency in this system & 16 & 1 & 1 & 0 & 0 \\
\hline 7. & I would imagine that most people would learn to use this system very quickly & 0 & 0 & 6 & 8 & 4 \\
\hline 8. & I found the system very cumbersome to use & 17 & 1 & 0 & 0 & 0 \\
\hline 9. & I felt very confident using the system & 0 & 1 & 0 & 9 & 8 \\
\hline 10. & I needed to learn a lot of things before I could get going with this system & 18 & 0 & 0 & 0 & 0 \\
\hline
\end{tabular}

Table 1. Results of the SUS questionnaire for volunteers below the 60 years threshold.

\begin{tabular}{|c|c|c|c|c|c|c|}
\hline & & $\begin{array}{r}1 \text { (Totally } \\
\text { disagree) }\end{array}$ & 2 & 3 & 4 & $\begin{array}{c}\text { 5(Totally } \\
\text { agree) }\end{array}$ \\
\hline 1. & I think that I would like to use this system frequently & 1 & 2 & 2 & 6 & 9 \\
\hline 2. & I found the system unnecessarily complex & 17 & 0 & 3 & 0 & 0 \\
\hline 3. & I thought the system was easy to use & 0 & 3 & 0 & 7 & 10 \\
\hline 4. & I think that I would need the support of a technical person to be able to use this system & 5 & 5 & 3 & 4 & 3 \\
\hline 5. & I found the various functions in this system were well integrated & 0 & 0 & 4 & 3 & 13 \\
\hline 6. & I thought there was too much inconsistency in this system & 15 & 4 & 0 & 1 & 0 \\
\hline 7. & I would imagine that most people would learn to use this system very quickly & 1 & 2 & 3 & 4 & 10 \\
\hline 8. & I found the system very cumbersome to use & 15 & 4 & 1 & 0 & 0 \\
\hline 9. & I felt very confident using the system & 0 & 0 & 6 & 8 & 6 \\
\hline 10. & I needed to learn a lot of things before I could get going with this system & 12 & 2 & 3 & 2 & 1 \\
\hline
\end{tabular}

Table 2. Results of the SUS questionnaire for volunteers above the 60 years threshold.

\section{Test phase and issues}

CoRe was first tested by 38 healthy subjects: 20 were above and 18 below the 60 -year age threshold, and had different education levels and degree of familiarity with a PC.

The system was tested with simulated rehabilitation sessions. After completing all the tasks, they were asked to fill in a Software Usability Scale (SUS)-based questionnaire (shown in Tables 1 and 2) and freely comment the system. During this phase some issues emerged, that lead us to look for new strategies and solutions to make CoRe as compliant to the patients' needs as possible.

It can be seen from the table that the subjects were more doubtful when asked about their capability and willingness to handle the system by themselves, without an expert by their side: for example, the answers for items \#4, \#7 and \#9 are less focused on the extreme values (that is, the ones that indicate total agreement/ disagreement with the statement) and more spread over the median ones, revealing a sort of "cautiousness" in the judgement. Considering other answers, according to which our system is not complex (\#2), inconsistent (\#6) or cumbersome to use (\#8), nor requires any previous knowledge to be used (\#10), it is clear how that "cautious" feedback is not a consequence of a defect of our system, but a symptom of the already mentioned uneasiness with technology. This hypothesis is confirmed by the analysis of each single subject's profile: median answers in the "critical" items match lower levels of familiarity with a pc and/or older age.

This relation between unease with technology and doubtfulness towards the system lead us to think that improving the involvement of the patients could reveal crucial to "distract" them and make them overcome their negative perception: this can be achieved by offering users a higher degree of immersion and entertainment. This lead to the identification of key upgrades to CoRe: continuous expansion of the stimuli database (to increase variability), as well as personalization (under the hypothesis that familiar stimuli - like pictures of relatives and references to known people/ situations - could be better accepted 
and more interesting than "default" stimuli available in a database) and introduction of 3D graphics.

The same version of CoRe used with the volunteers has also been tested by a small set of patients ( $n$ 1/4 15) from the IRCCS C. Mondino Foundation. The participants were supposed to undergo 12 sessions in a 1-month time range; these sessions were supposed to follow a well-defined protocol and required, for all the exercise types, the execution of a fixed number of instances of each available difficulty level. However, as mentioned in the 7 introduction, it is very difficult for all the patients to follow the same protocol. As a matter of fact, of the 15 subjects treated during the enrolment period, only 9, all afflicted by Parkinson's disease, were selected to be a part of the pilot study (an excerpt of their results is shown in Figure 5): the other 6 were rejected either for having undergone too few sittings before discharge, or (according to the therapists) for presenting a clinical picture too different from the majority of the samples to be comparable.

Of the 9 accepted, 6 patients performed all the sessions, while 3 were discharged before the end of the trial and dropped out after, respectively, 9, 10 and 11 sessions.

Considering that the SUS questionnaire is intended for use by "normal" subjects, it was impossible to administer it to the users involved in this part of the study, who are all affected by various degrees of cognitive impairments and thus needed the support of a specialist during the therapy. For this reason, the evaluation was based on a therapistprovided feedback, which resulted from their observation and interpretation of the patients' behaviour during the sessions. As a matter of fact, it must be noticed that, according to eligibility conditions, patients use CoRe in different ways: when no cognitive impairment is present, they may exercise autonomously, when it is present, they may need the help of a therapist (during visits) or of a home caregiver (during home treatment).

The most significant feedback obtained, beside a confirmation of the general appreciation and positive reaction to the system, was the clear preference for certain types of tasks. In particular, the subjects appeared more entertained and involved when they were asked to solve image-based exercises. When asked to solve a text-based exercise (e.g. "Unscramble the sentences" in Figure 6, left), it was not infrequent to hear them ask to switch to a visual exercise (e.g. "Unscramble the images" in Figure 6, right).

Since these patients were equally able to read words and recognize images, this confirmed that the main factor that triggered a patient's interest was not so much the task type as the "appealingness" of the implementation. For this reason, our optimization strategy included transforming textual tasks into image-based exercises whenever it was possible.

The first module to undergo the transformation was "Find the intruder". Contrary to other tasks in which grammar and syntax play a vital role, this one is based on recognition of concepts and thus better suited for visual reimagining. Among the two available solutions (the use of $2 \mathrm{D}$ or $3 \mathrm{D}$ visual elements), we chose the latter, for the reasons illustrated in "3D stimuli" section. Figure 7 shows the 3D version of that exercise.

\section{Discussion}

CoRe allows a therapist to easily generate a computerized version of exercises usually undergone with pen and paper during face-to-face rehabilitation sessions; besides, it records the patient's performance parameters during the execution of the session, so that 
the subject's assessment can be performed automatically by the system itself. This feature has two main consequences: first, it drastically reduces the time usually required to the therapist for the data analysis and, second, it introduces the option to automatically adjust the difficulty of the exercises based on the results of the assessment. The personalization of the exercises plays a vital role in preventing fatigue and boredom, since, as stated above, perceiving the rehabilitation as a stressful situation could very likely reduce the subject's compliance.

General Performance

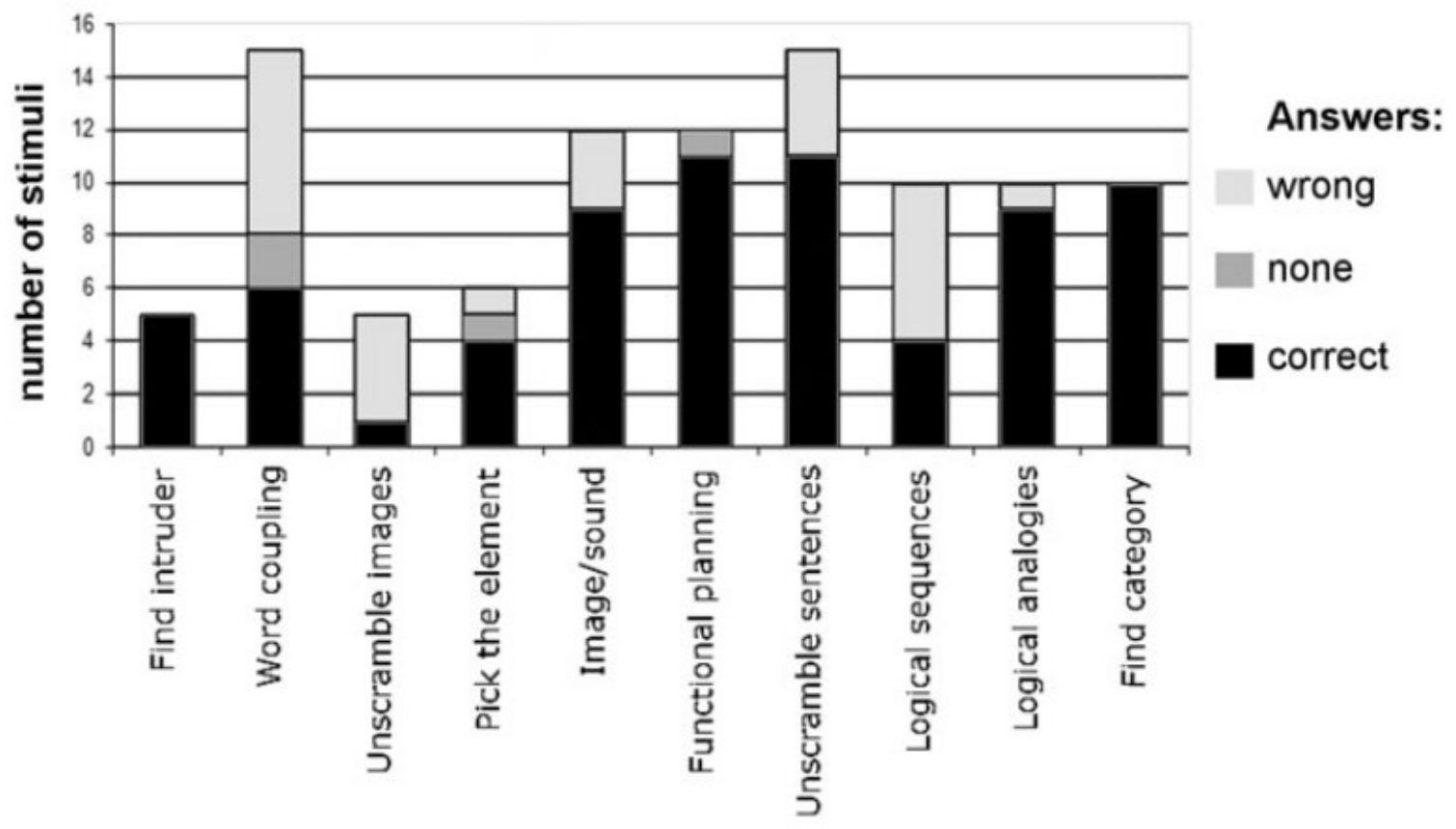

Figure 5. Bar chart representation of a patient's performance during a single session. Our system can also create pie charts for eachexercise and elaborate temporal trends in form of line charts.

Despite the first encouraging results, some aspects ofour system still need some improvements, especially those related to 3D features, and to the ontology update to meet further requirements for aphasic patients.

About the 3D aspect, the user is allowed to navigate freely in the virtual environment (a room with three bare walls and a floor, with a big table as the only piece of furniture, see Figure 7) to get as close to the objects as possible. This, coupled with the possibility to have a 360 \# view of the single objects, implied the need of a "complex" control system, since the simple combination of mouse buttons + keyboard arrows cannot fulfil all the required types of interaction. This is why another compromise is needed, this time between the degrees of freedom allowed to the user and the complexity of the controls. To solve this question, the introduction of particular hardware solutions must be taken into consideration: being the manipulation of objects a required feature, the use of a touch screen (maybe even a multi-touch for zooming purposes) could represent an interesting option. Of course, in that case usability tests must be undergone to verify whether this type of control is better accepted than the "classic" (even if slightly more complicated) mouse- 
keyboard combination.

In case of the default bare scenario not being well accepted by the users, it is also possible to recreate more familiar environments, just by adding textures to the walls and floor of the room. If this feature is accepted, it can also be used to generate different levels of difficulty for the exercise: for example, if the common category is "groceries", the use of a texture representing the aisles of a grocery store might help the patient pinpoint the context and, consequently, identify the intruder faster.
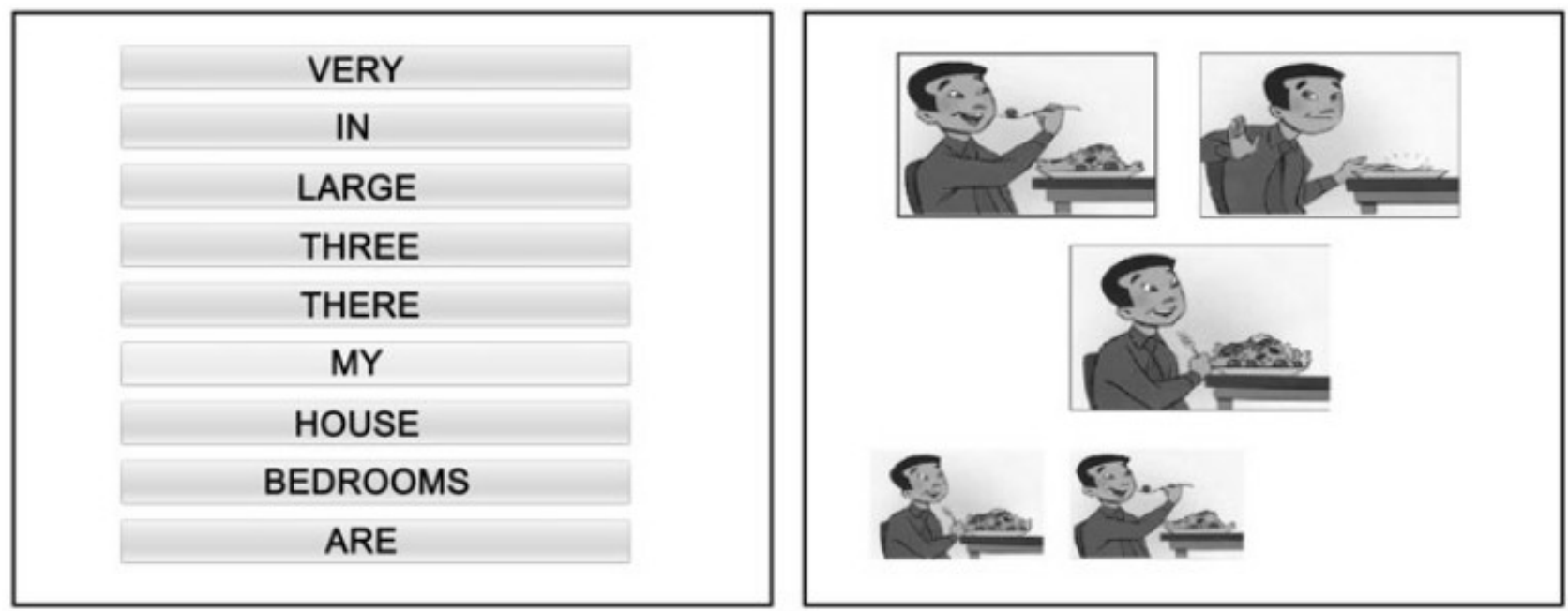

Figure 6. Instances of "Unscramble the sentence" and "Unscramble the images"

Also, it is not possible to assess which approach (text- only versus 2D versus 3D) will be preferred by every patient. Our goal is to offer, within one tool, the widest possible range of approaches from which to choose. This way the therapist will be able to test different options in the early phase of the treatment and decide which one represents the most suitable solution for the specific subject. In particular, this solution will be implemented within the homecare system.

Elderly patients (or, in general, people with little familiarity with modern technology) may well refuse the 3D approach in the beginning, and switch to it later, after acquiring familiarity with the computerized therapy, while younger individuals, accustomed to playing videogames, will very likely prefer undergoing 3D exercises from the beginning.

About the implementation of additional aphasia-related exercises, analyzing their structure it is clear how the future work will not just consist of implementation of GUIs and a simple software engine, but also a substantial upgrade of the ontology, to include new grammatical/semantic attributes (simple versus compound, word versus non-word, etc.) that allow relations to be created automatically and the dynamic generation of speechrelated exercises to happen. However, note that the generality of the ontology- based approach allows these updates occur without affecting the architecture of CoRe

\section{Conclusion and future work}

In this paper, we described the main features of CoRe, a computerized system for cognitive rehabilitation. Preliminary tests conducted on healthy volunteers and a small patients sample were aimed at evaluating the usability of the system and assessing which type ofstatistics may help therapists deciding how to continue the treatment. Results 
showed a good acceptance from patients and therapists. Once the 3D module will be completed and integrated with $\mathrm{CoRe}$, a new version of the system will undergo the test phase with the same set of volunteers enrolled the first time: this way, it will be possible to understand whether (and, if so, how) the enhancements we integrated in our tool influenced the users' perception of the tool itself.

Concerning the hardware support for CoRe, to date we limited the system for use on a PC, seen how our typical users are mostly elderly people who are often bad sighted and very likely also affected by reduced mobility. Considering the recent trend of creating smartphones and, in particular, tablets with larger screens, the idea of bringing the system to mobile devices could be taken into consideration, mostly for use cases involving younger patients.

Data collected so far do not support any conclusion about health and/or economic outcomes deriving fromthe systematic use of CoRe in the clinical routine. Thus, our intention is now to design clinical studies aimed at evaluating those outcomes.

\section{TRIANGLE}

\section{SPOON}

\section{HEXAGON}

\section{OCTAGON}

\section{CUBE}

Figure 3. An instance of "Find the intruder". Four stimuli belongto the geometric shapes class, the intruder belongs to kitchentools class

\section{Acknowledgements}

We wish to thank Marion Aumont, who collaborated with the creation of the 3D modules of the system.

\section{Declaration of interest}

The authors report no declaration of interest. 


\section{References}

1. Cappa SF, Benke T, Clarke S, et al. Cognitive rehabilitation. European Handbook of Neurological Management. Chichester, UK: Wiley-Blackwell; 2011;1:545-67.

2. Raymer AM, Patterson J, Cherney L, et al. Examining the effects of treatment intensity and constraint-induced language therapy for persons with stroke-induced aphasia: findings on an evidence-based systematic review. Arch Phys Med Rehabil 2007;88:e7.

3. Cappa SF, Benke T, Clarke S, et al. EFNS guidelines on cognitive rehabilitation: report of an EFNS task force. Eur J Neurol 2005;12:665-80.

4. Bean C. Techniques for enabling the older population in technology. $\mathrm{J}$ eLit 2004;1:109-21. Available from http://www.jelit.org/40/01/JeLit_17.pdf [last accessed 2 Oct 2014].

5. Cicerone KD, Dahlberg C, Kalmar K, et al. Evidence-based cognitive rehabilitation: recommendations for clinical practice. Arch Phys Med Rehabil 2000;81:1596-615.

6. Cicerone KD, Langenbahn DM, Braden C, et al. Evidence- based cognitive rehabilitation: updated review of the literature from 2003 through 2008. Arch Phys Med Rehabil 2011;92:519-30.

7. Cicerone $\mathrm{KD}$, Levin $\mathrm{H}$, Malec $\mathrm{J}$, et al. Cognitive rehabilitation interventions for executive function: moving from bench to bedside in patients with traumatic brain injury. J Cogn Neurosci 2006;18:1212-22.

8. Schuhfried. CogniPlus: I programmi di training. 1995. Available from: http://www.schuhfried.it/cogniplus-cps/i- programmi-di-training/[last accessed 25 Feb 2014].

9. Giunti OS. ERICA - Esercizi di Rlabilitazione Cognitiva per Adulti. 2010. Available from: http://www.riabilitazione.- giuntios.it/it [last accessed 25 Feb 2014].

10. Anastasis. COG.I.T.O. 2007. Available form http://cogito.integrazni.it /[last accessed 20 Aug 2014].

11. González-Ortega D, Díaz-Pernas FJ, Martínez-Zarzuela M, Antón-Rodríguez M. A kinect-based system for cognitive rehabilitation exercises monitoring. Comput Meth Progr Biomed 2014;113:620-31.

12. Fundación Intras - GRADIOR: software de Rehabilitación Neuropsicoógica del Deterioro Cognitivo. Available from: http://intras.es/index.php/productos/softwaregradior [last accessed 15 Sept 2015].

13. Fernández E, Bringas ML, Salazar S, et al. Clinical impact of RehaCom software for cognitive rehabilitation of patients with acquired brain injury. MEDICC Rev 2012;14:32-5.

14. Dwolatzky T, Whitehead V, Doniger GM, et al. Validity of the Mindstreams computerized cognitive battery for mild cognitive impairment. J Mol Neurosci 2004;24:33-44.

15. Kueider AM, Parisi JM, Gross AL, Rebok GW. Computerized cognitive training with older adults: a systematic review. PLoS One 2012. Available from: http://www.plosone.org/article/info\%3Adoi\%2F10.1371\%2Fjournal.pone.0040588 [last accessed 21 Feb 2014].

16. Tornatore JB, Hill E, Laboff JA, McGann ME. Self-administered screening for mild cognitive impairment: initial validation of a computerized test battery. $\mathrm{J}$ Neuropsychiatry Clin Neurosci 2005;17:98-105.

17. Eurostat. Computer use in individuals in EU countries. 2014. Available from: http://appsso.eurostat.ec.europa.eu/ nui/show.do?dataset 1/4 isoc_ci_cfp_cu\&lang $1 / 4$ en [last accessed 4 Oct 2014].

18. Eurostat. Individuals' level of computer skill in EU countries. 2014. Available from: http://epp.eurostat.ec.europa.eu/tgm/refreshTableAction.do?tab 1/4 table\&plugin 1/4 
1\&pcode $1 / 4$ t-sdsc460\&language $1 / 4$ en [last accessed 4 Oct 2014].

19. Richardson M, Zorn TE, Weaver K. Seniors' perspectives on the barriers, benefits and negative consequences of learning and using computers. 2002. Available from: http://www.academia.edu/download/31048190/resource1.pdf[last accessed 4 Oct 2014].

20. Alloni A, Tost D, Panzarasa S, et al. Enhancing computerized cognitive rehabilitation with 3D solutions. Proceedings of the 2nd ICTs for Improving Patient Rehabilitation Research Techniques Workshop - REHAB 2014; 2014; Oldenburg. Available from: http://eudl.eu/doi/10.4108/icst.pervasivehealth.2014.255358 [last accessed 29 Sep 2015]

21. Leonardi G, Panzarasa S, Quaglini S. Ontology-based automatic generation of computerized cognitive exercises. Stud Health Technol Inform 2011;169:779-83.

22. Abreu PF De, Werneck VMB, Costa RMEM Da, Carvalho LAV De. Employing Multiagents in 3-D game for cognitive stimulation. 2011 XIII Symposium on Virtual Reality. Uberlandia, Brazil; 2011 May 73-78.

23. Grau S, Tost D, Campeny R, et al. Design of 3D virtual neuropsychological rehabilitation activities. 2010 Second International Conference on Games and Virtual Worlds for Serious Applications; 2010 Mar. P 109-116. Available from: http://ieeexplore.ieee.org//pdocs/epic03/ wrapper.htm?arnumber 1/4 5459947 [last accessed 21 Jul 2014].

24. Rego P, Moreira PM, Reis LP. Serious games for rehabilitation a survey and a classification towards a taxonomy. 5Th Iberian Conference on Information Systems and Technologies (CISTI), 2010. Santiago de Compostela: IEEE; 2010.

25. Rego P, Moreira P, Reis LP. A survey on serious games for rehabilitation. 2010. Available from: http://paginas.-fe.up.pt/\#dsie10/presentations/session\%207/A \%20Survey\%20on\%20Serious\%20Games\%20for\%20Rehabilitation.pdf [last accessed 23 Jul 2014].

26. Tost D, Grau S, Ferre' M, et al. PREVIRNEC: a cognitive telerehabilitation system based on Virtual Environments. Haifa: IEEE. 2009:87-93. Available from http://ieeexplore.ieee.org/xpls/abs_all.jsp?arnumber=5174211\&tag=1 [last accessed 23 Jul 2014]

27. Rizzo A, Wiederhold BK. Virtual reality technology for psychological/neuropsychological/motor assessment and rehabilitation: applications and issues. IEEE Virtual Reality Conference (VR 2006); 2006 Mar 25-29. 308; Alexandria (VA). Available from: http://ieeexplore.ieee.org/xpl/ articleDetails.jsp? arnumber $=1667672$ [last accessed 22 Jul 2014] 